\title{
SUSAN BOYLE, LA FABULEUSE HISTOIRE DE LA TÉLÉVISION ET D'INTERNET
}

\author{
Sarah Sepulchre ${ }^{1}$
}

Début mai 2009, Visible Measures, une société spécialisée dans les mesures d'audience d'Internet, a publié une liste des vidéos les plus vues. En fait, leur The 100 Million Views Club - comme le nom l'indique - rassemble les séquences qui ont été regardées plus de 100 million de fois ${ }^{2}$. Ce jour-là, parmi les vingt vidéos les plus célèbres, huit sont des clips musicaux, quatre sont des bandes annonces de film, deux sont des extraits d'émissions télévisuelles. A ce moment, la prestation de Susan Boyle dans l'émission britannique, Britain's Got Talent, défrayait la chronique et faisait évidemment partie du classement. Seulement quatre films proposés par des internautes rivalisaient avec ces productions professionnelles. En conséquence, les observateurs ont rapidement formulé une hypothèse provocante : «Susan Boyle ou la défaite du Web $2.0^{3} »$.

Le Web 2.0 est normalement un lieu où l'internaute est roi. C'est le règne de l'interactivité, du partage de fichiers, du journaliste citoyen, de

1 Sarah Sepulchre est professeur au département de communication de l'UCL.

2 “The Visible Measures 100 Million Views Club", http://www.visiblemeasures.com/ the-100-million-club, consulté le 4 mai 2009.

3 G. NARVIC, "Susan Boyle ou la défaite du Web 2.0", http://novovision.fr/?SusanBoyle-ou-la-defaite-du-Web-2, consulté le 3 juillet 2009.

Recherches en communication, $\mathrm{n}^{\circ} 31$ (2009). 
You Tube et des blogs, du « many to many ». Le classement de Visible Measures - la vidéo de Susan Boyle en tête - démontre que l'utilisateur lambda n'est probablement pas aussi puissant qu'on pouvait le croire. En fait, celle qui semble tirer son épingle du jeu est surtout la télévision, celle-là même dont on prédisait la mort à cause de la toute puissance du "nouveau" média. « Pour la première fois de l'histoire, la télévision, ce vieux média, cette vénérable vieille dame a su utiliser le Web social et montrer sa puissance que l'on croyait endormie. Le plus grand mega hit buzzométrique de l'histoire est le fait d'une chaîne de télél. » Si la télévision, si les professionnels de l'audiovisuel sont ceux qui fournissent les films les plus consommés sur la Toile, il semblait logique de publier l'avis de décès du Web participatif. Des avis plus prudents ont presque simultanément nuancé cette affirmation. Cependant, Susan Boyle, son succès et le débat qui a suivi ont démontré que les "anciens" médias ne sont pas morts et que le "nouveau" n'a pas changé les pratiques autant qu'on pouvait le croire.

Finalement, il y a longtemps qu'on sait qu'un "nouveau" média ne tue pas le précédent. "A chaque émergence d'un "nouveau" média, on annonce la mort du précédent. Selon McLuhan, la télévision allait révolutionner le monde, apporter les connaissances via le son et l'image, créer une tribu mondiale. Aujourd'hui, la télévision est critiquée de partout $^{2}$. » Les médias ne se remplacent pas, mais s'accumulent. On ne va pas y revenir dans cet article. Ce qui nous intéresse dans la célébrité virale de Susan Boyle, c'est l'éclairage particulier qu'elle offre à la notion de « re-médiation » de Jay David Botler et Richard Grusin.

Jan Baetens explique la « re-médiation » comme « la transformation d'un média par/en un autre ${ }^{3} »$. Jay David Bolter et Richard Grusin considèrent qu'il s'agit d'une caractéristique majeure des "nouveaux" médias. « [W]e call the representation of one medium in another remediation, and we will argue that remediation is a defining characteristic of the new digital media ${ }^{4}$. " Jan Baetens s'attarde particulièrement au

1 F. EPELBOIN, "Susan Boyle ou l'avènement du Web 2.5", http://owni.fr/2009/05/02/ susan-boyle-avenement-Web, consulté le 3 juillet 2009.

2 E. MAIGRET, Sociologique de la communication et des médias, Colin, Paris, 2003, p. 258.

3 Cette citation est reprise de l'artice précédent de J. Baetens.

4 J. D. BOLTER et R. GRUSIN, Remediation. Understanding New Media, The MIT 
concept de « repurposing » (que l'on pourrait traduire par « réappropriation », nous dit-il), un type de « re-médiation »: «[...] les auteurs soulignent la possibilité ouverte à chaque "ancien" média de survivre en adoptant certains aspects d'un média plus récent (que l'on pense par exemple au roman, "remédié" par le cinéma mais capable de se rajeunir en embrassant des manières de raconter plus visuelles et pour tout dire plus cinématographiques $)^{1}$. \ Jan Baetens ajoute que, selon les deux auteurs, il est impossible d'isoler un média, ils sont toujours en contact et s'influencent donc mutuellement. « Media are continually commenting on, reproducing, and replacing each other, and this process is integral to media. Media need each other in order to function as media at $\mathrm{all}^{2}$. » La « re-médiation » permet donc de lire les évolutions concommitantes des "anciens" et "nouveaux" médias, leurs rapprochements, leurs oppositions, leurs ressemblances et leurs divergences.

Le propos de cet article est d'examiner l'évolution d'Internet et des médias traditionnels à travers cette notion de remédiation. Dans un premier temps, nous allons examiner les caractéristiques de ce "nouveau média”. Jean-Françosi Fogel et Bruno Patino considèrent qu'il s'agit de l'instantanéité, de la personnalisation et de l'interactivité. Nous montrerons ensuite deux évolutions parallèles du monde médiatique - d'un côté l'adaptation des anciens médias; de l'autre la remise en question de la révolution Internet - qui semblent confirmer les thèses de Jay David Bolter et Richard Grusin.

\section{La révolution Internet}

En 2007, Jean-François Fogel et Bruno Patino s'intéressaient à la situation de la presse sur Internet. Ils proclamaient : « Internet n'est pas un support de plus ; c'est la fin du journalisme tel qu'il a vécu jusqu'ici. [... La presse n'a pas entamé un nouveau chapitre de son histoire, mais bien une autre histoire, sous le régime d'Internet ${ }^{3}$. " Sans avoir une perception idyllique ni catastrophiste du "nouveau" média, les auteurs insistaient sur la rupture radicale qu'il représentait. Cette révolution

Press, Cambridge, 2000, p. 45.

1 Cette citation est reprise de l'artice précédent de J. Baetens.

2 J. D. BOLTER et R. GRUSIN, op. cit., p. 55.

3 J.-Fr. FOGEL et B. PATINO, Une presse sans Gutenberg, Grasset, Paris, 2005, pp. 11-13. 
peut être examinée à partir de quelques caractéristiques du média : l'instantanéité, les capacités de personnalisation, l'interactivité.

Il suffit de surfer sur quelques sites pour se rendre compte qu'Internet est le média de l'instantanéite. Cela se vérifie par rapport à l'information évidemment, mais pas uniquement. L'annonce du décès de Michael Jackson n'était pas encore confirmée par les autorités, ni l'hôpital qui avait pris en charge le chanteur, qu'à 16 heures trente le 25 juin 2009,TMZ1 répandait déjà la nouvelle. Il suffit de quelques fractions de secondes à un moteur de recherche pour trouver le site que l'on cherche. En quelques clics, on peut regarder un film, écouter une chanson, réserver une chambre d'hôtel. "Internet, dernier né des médias, est souvent présenté comme le média de la vitesse, "disponibles 24 heures sur 24, sans frontières géographiques [qui] offre la possibilité de faire le tour de la planète en quelques secondes et de l'utiliser sans autres limites que celle de notre imagination" (Ettighoeffer, Blanc, 1998, p. 138)2.»

La Toile bouscule également le rapport au temps qu'avaient institué les médias traditionnels : l'immédiateté de la radio, le rendezvous programmé de la télévision, le bouclage tardif de la presse écrite ou la lente régularité des magazines. Sur Internet, n'importe qui peut annoncer n'importe quoi en quelques minutes. La plupart des sites comportent aujourd'hui un bloc «news » constamment actualisé sur leur page d'accueil, qu'il s'agisse des plateformes en ligne des "anciens" médias, mais aussi des pages de groupes musicaux, d'entreprises, etc.

En 1996, le Wall Street Journal lance une édition Web où l'abonné peut sélectionner les rubriques qui l'intéressent. Aujourd'hui, Amazon enregistre les adresses IP et la navigation des utilisateurs. Cela lui permet de personnaliser ses propositions d'achats en fonction du profil de chaque internaute. Sur la plupart des sites, les lecteurs peuvent enregistrer leurs paramètres personnels ou introduire des critères qui orientent les services proposés en fonction de leur personnalité, de l'endroit de leur domicile, de leurs préférences, etc. A côté de cette personnali-

1 "Michaël Jackson - Cardiac Arrest", http://www.tmz.com/category/michaeljackson/page/12/, consulté le 6 juillet 2009.

2 Ch. DELEU, "Médias et nouvelles technologies : le journaliste pressé", Les Cahiers du journalisme, $\mathrm{n}^{\circ} 5$, décembre 1998, p. 213. 
sation des contenus, il faut également souligner que chaque internaute poursuit un chemin différent, de mot-clé en mot-clé, d'hyperlien en hyperlien. «Internet est un média que l'internaute bâtit à chaque visite et de façon d'autant plus subjective qu'il ignore ce qu'il délaisse ${ }^{1}$. » Le public d'Internet est donc fragmenté, émietté dirait Dominique Wolton². « Des pages, des pages, des pages produites à la demande, par un algorithme qui ne répètera plus la même combinaison, et qui sont vues par un internaute qui en constitue l'audience à lui tout seul's. »

Jean-François Fogel et Bruno Patino explorent le contexte médiatique qui a entouré les trois attentats perpétrés par Al-Qaeda (le 11 septembre 2001 à New York, le 11 mars 2004 à Madrid, le 7 juillet 2005 à Londres). En 2001, les médias traditionnels battent des records d'audience alors que les sites Internet subissent un échec complet. Soit ils sont en panne parce qu'ils n'ont pas pu gérer le nombre d'internautes qui souhaitaient se connecter, soit ils offrent quelques textes, quelques photos rapidement dépassés par le poids des images télévisuelles. En 2004, le trafic est devenu fluide et les sites proposent des contenus multimédia (des cartes, des graphiques, etc.). Mais c'est en 2005, qu'Internet prend toute sa dimension. Le contenu des sites est désormais alimenté par les citoyens et non uniquement par les journalistes. Les victimes utilisent leurs portables pour prendre des photos ou des vidéos et pour envoyer des témoignages.

L'interactivité est la caractéristique essentielle du Web 2.0 et elle dépasse largement ce qui existait auparavant (principalement le courrier des lecteurs). L'interactivité débute dans les blogs qui possèdent une fonction de commentaire. Depuis, la technique a migré vers toutes les pages. Dans cette conception du Web, chacun est à la fois émetteur et récepteur. Au-delà du simple dialogue, on assiste à la naissance d'une véritable écriture conjointe sur Internet. Analysant le journalisme en ligne, Sandro Faes et Emmanuel Murhula remarquent que « [...] l'information médiatisée se trouve ainsi co-construite au sein d'un nouveau cadre où informations formelles et informelles se côtoient, se répondent et se bousculent ${ }^{4}$. » Selon Jean-François Fogel et Bruno Patino, cela

1 J.-Fr. Fogel et B. Patino, op. cit., p. 63.

2 D. Wolton, cité par E. MAIGRET, op. cit., p. 267.

3 Idem, p. 170.

4 S. FAES, E. MURHULA, "La liberté d'informer retrouvée", dans A. Klein (dir.) 
remet en question la notion même d'auteur. Quand un lecteur répondait à un média traditionnel, cela ne changeait pas l'information. Le contenu restait immuable, précisent-ils. Sur Internet, la « réponse rejoint directement le contenu qui l'a déclenchée. [...] l'information se modifie au fur et à mesure qu'elle circule ${ }^{1}$. » En effet, internaute et auteur du site possèdent des moyens équivalents, sur la forme (ils peuvent tout deux envoyer des écrits, des images fixes ou animées) et sur le fond (puisque l'information informelle est mise sur pied d'égalité avec celle provenant des professionnels de la communication). L'interactivité est donc totale.

Compte tenu de ces changements, nous l'avons dit, Jean-François Fogel et Bruno Patino proclamaient que les médias étaient entrés dans une nouvelle ère tant Internet dépassait, transcendait les "anciens" médias. Avec la Toile, l'écrit perd son statut de référence pour devenir un signal comme les autres affiché sur un écran. L'écoute collective qui incombait aux radios et télévisions et qui créait le lien social grâce au rendez-vous fixes de la grille (le journal télévisé à 20 heures, par exemple) n'a plus de raison d'être. Bref, selon les deux auteurs, Internet rend inintéressants les traits dominants des médias de masse. «D'un site à l'autre, tous les contenus défilent sur le même réseau, avec un label unique : disponible en ligne. Sur l'écran, les spécificités de chaque presse deviennent de simples variations d'une seule expérience, le journalisme diffusé par Internet ${ }^{2}$. » Autant annoncer la mort des "anciens" médias.

\section{La contre-attaque de la télévision}

Et pourtant, l'effervescence autour de Susan Boyle montre bien que l'enterrement de la télévision (mais on pourrait étendre la réflexion à tous les médias traditionnels) n'est pas si imminent que cela. La télévision s'est adaptée de deux manières : d'abord, elle a apprivoisé le Web; ensuite, elle a changé ses pratiques.

Les chaînes ont toutes un portail Internet sur lequel elles exploitent toutes les possibilités du média. Elles ont intégré la notion d'ins-

Objectif blogs!, L'Harmattan, Paris, 2007, p. 70.

1 J. Fr. FOGEL et B. PATINO, op. cit., pp. 146-147.

2 J. Fr. FOGEL et B. PATINO, op. cit., p. 22. 
tantanéité. Sur la page d'accueil de la plupart d'entre elle, on trouve des informations continuellement actualisées. Certaines lient d'ailleurs la page d'accueil Internet au programme qui passe au même moment sur le téléviseur. La personnalisation est également favorisée. Sur le site de $R T L$, il est par exemple possible d'enregistrer le lieu de son domicile pour obtenir des prévisions météorologiques locales ${ }^{1}$. On peut également s'inscrire afin «d'accéder à tous les contenus personnalisés de RTL info ", " d'accéder à un espace personnalisé pour gérer votre profil », de « bénéficier des newsletters, mais également d'information sur les programmes de RTL-TVI et BEL $\mathrm{RTL}^{2}$ ». L'interactivité est également utilisée par les chaînes. La plupart des contenus peuvent être commentés. On peut poser des questions générales ${ }^{3}$ ou aux animateurs ${ }^{4}$, on peut participer à des concours ou prolonger le contenu des émissions par des discussions sur des forums ${ }^{5}$, on peut participer aux émissions en posant des questions par mail, voire par webcam comme pour l'émission Controverse $e^{6}$. Les chaînes de télévision ont également tiré parti de l'extraordinaire capacité de stockage qu'offre maintenant Internet et proposent de visionner les anciennes émissions. TF1 permet de revoir les meilleurs moments d'une émission ou un programme en intégralité. Elle propose 16500 vidéos issues des grilles actuelles, mais aussi des archives depuis $1980^{7}$.

La télévision a donc tiré parti d'Internet, elle a aussi changé ses pratiques. Sur la page de l'émission Toute une histoire de Jean-Luc Delarue, on peut trouver des bonus (des vidéos des coulisses principalement), le "chat" de la psychologue qui intervient dans le programme. Après l'émission télévisuelle, l'enregistrement continue et ce prolongement n'est visible qu'en ligne ${ }^{8}$. Les programmes, désormais, ne sont plus conçus comme des émissions diffusées une seule fois à un horaire fixe pour un public passif. La télévision sait qu'elle doit prévoir des

1 http://www.rtlinfo.be/index.php, consulté le 6 juillet 2009.

2 http://www.rtlinfo.be/rtl/user/register, consulté le 10 août 2009.

3 http://www.tf1.fr/tf1-et-vous, consulté le 6 juillet 2009.

4 http://www.tf1.fr/tfl-et-vous/rendez-vous-avec/laurence-ferrari, consulté le 6 juillet 2009.

5 http://forums.france2.fr/france2/envoyespecial/liste_sujet-1.htm, consulté le 6 juillet 2009.

6 http://www.rtltiv.be/controverse, consulté le 6 juillet 2009.

$7 \mathrm{http} / / /$ videos.tf1.fr/, consulté le 6 juillet 2009.

8 http://toute-une-histoire.france2.fr/?page=accueil, consulté le 6 juillet 2009. 
produits qui seront disponibles sur différents supports, de manière continue, qui pourront être prolongés par des dossiers de textes, de photos ou de vidéos. Surtout, elle s'adresse à des publics fragmentés, prêts à participer, habitués à picorer des programmes de ci de là au fil de ses navigations. La télévision s'est donc adaptée, confirmant l'hypothèse de Jay David Bolter et Richard Grusin selon laquelle un média traditionnel survit en adoptant certains aspects d'un média plus récent (cfr. supra).

\section{Internet, le putsch manqué}

Mais Internet est-il la révolution qu'on attendait ? Le premier contenu d'un "nouveau" média est le média précédent selon Marshal McLuhan. La formule s'applique aussi à ce "nouveau" média. D'abord parce qu'y trouve les médias traditionnels. Cinéma, télévision, presse écrite, radio possèdent tous un portail en ligne. Il est vrai que, quand on cherche une information, on se rend d'abord sur Google News, mais le site renvoie... vers Le Figaro, Le soir, L'Express, etc. Et ce sont ces sites qui comptent un nombre important de visites. En effet, selon une étude de The Diffusion group citée par Numerama, les vidéos d'internautes devraient représenter $42 \%$ des vidéos regardées sur Internet, mais elles ne rassemblent que $4 \%$ des recettes. De leur côté, les vidéos professionnelles, les $58 \%$ restant, génèrent $96 \%$ des revenus. Et les revenus sont proportionnels à la célébrité.

Ensuite, il faut bien constater qu'Internet n'innove pas tant que cela. C'est vrai, les "anciens" médias ont dû adapter leur présentation et leur manière d'écrire. C'est vrai, on a vu apparaître des blogs, des réseaux en ligne, Twitter, etc. Mais finalement, la musique qu'on écoute sur Deezer, c'est Nirvana, Johnny Halliday et Madonna. Les romans qu'on lit en ligne sont ceux de Stephen King. Les séries qu'on charge sont celles des grandes chaînes américaines. Il existe évidemment des créations sur Internet. Des auteurs y publient leur texte (notamment les poètes dont parlait Jan Baetens), des groupes diffusent leur musique (notamment sur Myspace), des réalisateurs dévoilent leurs films (sur Youtube), des Web radios sont lancées. Cependant, ces sites ne connaissent pas (encore) la célébrité des sites plus « grand public ». L'exemple des Web séries est intéressant à ce propos. Des internautes proposent des productions sérielles propres sur le Net. La qualité varie fortement de l'une à l'autre et elles connaissent des fortunes diverses. Mais elles 
restent confidentielles. Quand elles ont du succès, c'est parce qu'elles sont la création des acteurs traditionnels des médias. The Marvellous Flying Box de Michael Havenith est interprété par des acteurs de théâtre et est hébergé sur le site d'un quotidien populaire belge1. Depuis avril 2009, Nous ne sommes pas des saints est la première Web série à être adaptée par une chaîne de télévision en France ${ }^{2}$. Mais elle était produite par des professionnels de l'audiovisuel. Son créateur est Nicolas Ragni, qui a mis les premiers épisodes en ligne alors qu'il démarchait les chaînes pour la vendre ${ }^{3}$.

« Si l'on distingue l'insertion effective d'Internet dans les pratiques sociales des utopies technicistes et si l'on analyse les dispositifs employés, il apparaît clairement qu'Internet n'est pas la révolution annoncée. Ceci ne signifie pas que son apport doive être négligé et son impact social sous-estimé, simplement que le changement économique et technique, pour majeur qu'il soit, ne bouscule pas de lui-même les rapports humains en ce début de siècle ${ }^{4} \gg$, dit Eric Maigret. Bref, Internet ne semble pas (ou pas encore) être le séisme qu'on prévoyait. Il offre effectivement une plateforme nouvelle aux "anciens" médias, mais il ne produit pas encore assez de contenu propre qui pourrait faire le poids. Des craintes laissaient croire qu'il pourrait devenir un « supermédia ${ }^{5}$ », un média qui aspirerait tous les autres. Cependant, on remarque que la télévision, les journaux, les magazines, les radios traditionnels existent toujours. D'un autre côté, on voit qu'Internet a fortement influencé les "anciens" médias. Ils ont compris l'intérêt de la personnalisation, de l'interactivité, de l'instantanéité et les ont adaptés pour leurs supports respectifs. Ils se ne se conçoivent plus comme des médias exploitant un support unique à destination d'un public homogène. L'apport d'Internet ne doit donc pas être négligé.

1 The Marvellous Flying Box, sur La Dernière Heure, http://tmfb.dhnet.be, consulté le 7 juillet 2009.

2 http://www.nousnesommespasdessaints.com/accueil.php, consulté le 7 juillet 2009.

3 E. Cario, “Nous ne sommes pas des saints', série paradisiaque”, http://www.ecrans. fr/Nous-ne-somme-pas-des-saints-serie,2899.html, consulté le 7 juillet 2009.

4 E. MAIGRET, op. cit., p. 258.

5 E. MAIGRET, op. cit, p. 256. 


\section{Retour à la notion de « re-médiation »}

La « re-médiation » de Jay David Bolter et Richard Grusin semble parfaitement s'appliquer à nos "anciens" et "nouveaux" médias. La télévision s'est bien adaptée pour survivre. L'Internet recycle véritablement les "anciens" médias. Plus encore, il paraît évident que les différents médias sont bien en contact constant et s'influencent mutuellement. La théorie de la « re-médiation » permet donc de sortir complètement de l'idée de succession des médias, de leur mort quand un nouveau apparaît, pour la remplacer par l'idée de co-existence et de co-influence.

Les commentateurs des nouvelles technologies, discutant du cas de Susan Boyle, en sont arrivés à une conclusion similaire de celle de Jay David Bolter et Richard Grusin. Quelques jours après l'apparition de Susan Boyle sur le Web, Guilleaume Narvic propose une lecture du phénomène. Il remarque alors que la véritable nouveauté de l'événement n'est pas dans l'histoire en elle-même (la revanche des laides comme certains l'ont qualifiée), n'est pas non plus dans l'ampleur du buzz ( « car tout de même il n'a pas encore atteint celle du premier pas sur la Lune $^{1}$ ! », dit-il), mais se situe dans le fait que les télévisions en ont parlé sans souligner les dangers d'Internet ou la peur de la concurrence déloyale qui étaient pourtant ses thèmes favoris concernant le réseau. L'affaire Susan Boyle marque la fin de la guerre entre "anciens" et "nouveaux" médias. Guilleaume Narvic souligne qu'ils ne s'opposent pas. " Au contraire, ils se renforcent ! C'est même leur conjonction, leur... hybridation, le fait qu'ils se répondent l'un l'autre, dans un véritable dialogue, en démultipliant l'effet viral, qui permet ce phénomène inédit. Le Web 2.0 s'est trompé. Complètement. Nous ne sommes pas du tout dans une logique d'opposition et de substitution, mais bien dans une logique d'hybridation (la collision des galaxies)2. "

Finalement, c'est un peu ce que dit Dominique Wolton quand il affirme que c'est la complémentarité entre les médias de masse et Internet qui crée du lien social.

1 G. NARVIC, "Susan Boyle (2) : quand la télévision se trouve si belle dans le miroir d'Internet", http://novovision.fr/?Susan-Boyle-2-quand-la-television, consulté le 6 juillet 2009.

2 G. NARVIC, "Susan Boyle ou la défaite du Web 2.0", op. cit. 
Un média de la demande, Internet, est toujours plus fort qu'un média de l'offre car, par définition, il vous apporte ce que vous voulez. Mais il est aussi plus faible car vous n'allez chercher que ce que vous voulez. Or, un citoyen doit s'intéresser à ce qui lui plaît mais aussi à ce qui ne l'intéresse pas du tout. Donc, seul le média de l'offre l'oblige à sortir de ses intérêts personnels pour aller vers la collectivité. Non seulement les deux sont complémentaires mais plus il y a des médias de la demande individualisés, comme Internet, plus le média de l'offre est nécessaire, simultanément et même préalablement ${ }^{1}$.

L'hypothèse formulée par Jay David Bolter et Richard Grusin, Guilleaume Narvic ou Dominique Wolton est, qu'après quelques années de déstabilisation, le système médiatique va probablement retrouver un équilibre dans lequel tous les supports auront une place et où ils s'influenceront les uns les autres. Il faudra attendre afin de déceler si ce sont les visions défaitistes ou optimistes qui auront raison.

1 D. WOLTON, McLuhan ne répond plus. Communiquer c'est cohabiter. Entretiens avec Stéphane Paoli et Jean Viard, Editions de l'Aube, 2009, p. 16. 